\title{
Smart Products in der Logistik: Unterstützung von Mehrwertdienstleistungen durch Wearables
}

Sebastian Werning, Tobias Mollen-Ungru, Dennis Konusch und Ingmar Ickerott

Der Bedarf an Zusatzleistungen in der Logistik-Branche nimmt zu. Value Added Services (VAS) verhelfen dem Logistikdienstleister dazu, Diversifikation zu erlangen und sich am Markt von anderen Logistikdienstleistern abzuheben. In diesem Beitrag werden anstatt klassischer Logistik-Serviceleistungen, die oftmals an Dritte ausgelagert werden, moderne Technologien für die Erbringung kundenindividueller Zusatzleistung betrachtet. Diese sollen weitere VAS in der Praxis ermöglichen. Mithilfe von Pilotprojekten, die im Rahmen von Kooperationen zwischen der Hochschule Osnabrück und einem Logistikdienstleister aus der Fashion-Branche stattgefunden haben, konnten klassische VAS definiert werden. Nach Durchführung einer systematischen Literaturrecherche hinsichtlich des Einsatzes von Wearables, insb. der Smart Glasses, konnten mehrere Einsatzgebiete kategorisiert werden, in denen neuartige Technologien einen Mehrwert in logistische Prozesse einbringen und somit neue VAS-Potenziale aufzeigen. Die Relevanz dieses Beitrags zeichnet sich einerseits durch die Implikation für die Forschung hinsichtlich des Einsatzes von intelligenten Technologien aus, um VAS zum Nutzen eines Logistikdienstleisters anwenden zu können. Andererseits werden mögliche Einsatzgebiete für Smart Glasses zur Erbringung von VAS in der Logistik vorgestellt und technische als auch ergonomische Herausforderungen betrachtet.

\section{Einleitung}

Das Geschäftsmodell der Kontraktlogistik unterscheidet sich signifikant von den Wertschöpfungsmodellen anderer Branchen im Produktions- und Dienstleistungssektor. Der Unterschied besteht in der Verknüpfung klassischer logistischer Tätigkeiten mit ergänzenden Sondertätigkeiten, die häufig eher dem Produktionssektor zuzuschreiben sind. Kennzeichnend für die kontraktlogistischen Prozesse ist dabei stets die Eigenschaft, dass sämtliche Wertschöpfungsstufen ausschließlich im Rahmen konkreter Kundenaufträge zu erbringen sind. Bei der Ausgestaltung dieser Kombination aus Produktion und klassischer Dienstleistung im Kundenauftrag entsteht das weite Feld der sog. Zusatzdienstleistungen oder Value Added Services (VAS). Mit diesem Begriff ist ein konkretes Verständnis verbunden, welches 
in diesem Beitrag näher beschrieben wird. Das Thema Zusatzdienstleistung wird dazu zunächst von dem logistischen Kernprozess abgegrenzt. Anschließend können VAS noch anhand gängiger Unterscheidungskriterien kategorisiert werden. Innerhalb dieser Kategorien seien die Herausforderungen und die Möglichkeiten zur Unterstützung von Tätigkeiten durch den Einsatz von Wearables, insb. Smart Glasses beleuchtet. Die Analyse der Unterstützungsmöglichkeiten beschränkt sich dabei nicht auf die Möglichkeiten aktuell am Markt verfügbarer Technologieträger. Vielmehr seien unter der Annahme technologischer Verbesserungen der bestehenden Systeme auch denkbare Verfahren beschrieben, für die der Einsatz von Wearables und VAS sinnvoll erscheint. Hierfür werden in diesem Beitrag Anwendungsfälle für den Einsatz von Smart Glasses zur Realisierung von VAS in der Logistik-Branche identifiziert und klassifiziert. Anhand der sich ergebenden Übersicht können Unternehmen in der Praxis bestimmen, ob der Einsatz von Smart Glasses für ihre Geschäftsprozesse in Frage kommt. Darüber hinaus erlaubt die Beschreibung der Anwendungsfälle eine gezielte Umsetzung für das eigene Unternehmen.

\section{Value Added Services im Geschäftsmodell der Kontraktlogistik eines Fashion-Unternehmens}

Wie auch in anderen Fachdisziplinen ist die Suche nach einer einheitlichen Definition der Logistik mit Herausforderungen verbunden, da die führenden Autoren der Fachdisziplinen die Aufgabenschwerpunkte, die der Logistik zugewiesen werden, unterschiedlich gewichten (Huth 2015; Ehrmann 2017). Vor allem die Betrachtung eines bestimmten Unternehmens aus der Fashion-Branche stellt einen sehr praxisbezogenen Horizont dar. Das Unternehmen wird im Laufe dieses Abschnitts näher beleuchtet. Theorie und Praxis vereinen sich in diesem Bezug auf die wesentlichen Aspekte der Logistik. So beinhaltet die Logistik die reibungslose Gestaltung des Material-, Wert- und Informationsflusses, die sowohl in Fachliteratur aufgegriffen werden kann als auch als Grundgerüst für Logistikprojekte aus der Praxis dient (Ehrmann 2017).

Zwecks Eindeutigkeit innerhalb dieses Beitrages soll der Logistikbegriff nach Ehrmann wie folgt herangezogen werden. Die Logistik leitet planerische und ausführende Maßnahmen aus den Zielen eines Unternehmens ab. Zur Gewährleistung eines optimalen Material-, Wert- und Informationsflusses werden einzusetzende Instrumente definiert und in den betrieblichen Leistungserstellungsprozess integriert. Dieser erstreckt sich von der Beschaffung von Produktionsfaktoren und Informationen über deren Bearbeitung und Weiterleitung bis zur Distribution von erstellten Leistungen (Ehrmann 2017). Anhand dieser Definition lassen sich Einsatzschwerpunkte für die Logistik ableiten, in folgende Logistikanwendungen zusammenfassen, wobei zu beachten ist, dass die genannten Aufgaben nicht als isolierte Teilaufgaben, sondern als ein zusammenwirkendes und einheitliches logistisches Konzept zu verstehen sind (Ehrmann 2017): Beschaffungslogistik, Lagerlo- 
gistik, Produktionslogistik, Transportlogistik, Ersatzteillogistik, Instandhaltungslogistik, Distributionslogistik, Entsorgungslogistik, Informationslogistik und Branchenlogistik.

Die steigende Nachfrage nach kundenindividuellen und kundenorientierten Lösungen zwingt Logistikunternehmen dazu, neben den obigen Hauptaufgaben zusätzliche wertschöpfende Zusatzleistungen anzubieten (Meffert 1994). Diese wertschöpfenden (Mehrwert-)Dienstleistungen, engl.: value added services (VAS), ergänzen somit die Hauptaufgaben von Logistikunternehmen (Frohn 2006). Diese Entwicklung ermöglicht es Logistikunternehmen, sich als Marketinginstrument nicht nur auf das Preis-Leistungs-Verhältnis zu fokussieren (markttypisch), sondern das Angebot von wertsteigernden Zusatzlösungen in den Mittelpunkt ihres Marktauftrittes zu stellen (Davies et al. 2006). Um Kunden bemerkenswerte Mehrwerte bzw. Differenzierungsmerkmale zu verschaffen und sich am Markt durchzusetzen, können diese wertschöpfenden Dienstleistungen im Angebotsportfolio der Logistikunternehmen bereitgestellt werden. Kunden werden als Nutzer der Dienstleistungen eines Logistikunternehmens verstanden. Die angebotenen Leistungen werden meist mit den Kerngeschäftsaufgaben des Unternehmens kombiniert und in einem Leistungspaket angeboten. Aus Kundensicht sind VAS somit ausgelagerte Dienstleistungen und Aktivitäten, die ein Dritter Unternehmer übernimmt (engl.: outsourcing) (Laakmann 2018).

Der Begriff Mehrwertdienstleistungen oder VAS wird in verschiedenen wissenschaftlichen Fachrichtungen unterschiedlich definiert. Im Marketing bspw. wird die Einordnung dieser wertsteigernden Tätigkeiten in das Konzept des „Value Added Marketing" vorgenommen und bezieht sich dort auf die Summe der Dienstleistungen, welche die Kernleistung eines Angebots wertsteigernd anreichern. Die Definition stammt aus der Perspektive des Kunden. Der VAS dient dem Zweck einen nachvollziehbaren und akzeptierten Mehrwert zu generieren, der über die originären Produkteigenschaften hinausgeht, um bei homogenen Konkurrenzangeboten einen Wettbewerbsvorteil zu erreichen (Bongartz 2018).

Im Kontext der Kontraktlogistik greift diese Definition jedoch zu kurz. Hier wird der Mehrwert für den Kunden nicht (allein) über eine Ergänzung der Produkteigenschaften im Verhältnis zu Konkurrenzangeboten geschaffen. Vielmehr handelt es sich häufig um einen Katalog von Tätigkeiten, welche um logistische Dienstleistungen erweitert werden können. Eine Tätigkeit kann als ein Service bezeichnet werden und hat zum Ziel, notwendige Basisanforderungen von Folgeprozessen im Kundenumfeld abzudecken. Gerade im B2B-Geschäft sind hier die logistischen und warenwirtschaftlichen Prozesse auf Kundenseite maßgeblich (Zimmerlich et al. 2005).

Aus diesem Grund fasst das Fraunhofer Institut für Materialfluss und Logistik eine spezifische Definition des Begriffs „Value Added Services“. Dies sind wertschöpfende (Mehrwert-)Dienstleistungen, die nicht zu den Haupttätigkeiten (wie bspw. den Transport und Umschlag von Gütern) durch einen Logistikdienstleister gehören. VAS können originär logistischer Art, wie z.B. Transportverpackung 
und Verwiegung, oder auch originär nicht-logistischer Art sein. Darunter fällt bspw. die Finanz- oder Rechnungswesenleistung (Fraunhofer-Institut 2006).

\subsection{Kern- vs. Zusatzfunktionen in der Lagerlogistik: Einordnung der Mehrwertdienstleistungen anhand der VDI Richtlinie 3601}

Im Rahmen des Projektes GLASSHOUSE wurden vorwiegend die originär logistischen Dienstleistungen im Hinblick auf eine mögliche Unterstützung durch Wearables untersucht. In diesem Segment ergibt sich ein umfangreiches Spektrum möglicher Ansätze für Sondertätigkeiten. Die Vielfalt der möglichen Einsatzszenarien für ein Smart Product als Wearable zu erkennen, gelingt durch die Einordnung in die VDI Richtlinie 3601. In dieser Richtlinie sind die Kern- und Zusatzfunktionen eines Warehouse-Management-Systems (WMS) verdeutlicht. Somit wird zunächst Stellung zur Grundlage der Mehrwertdienstleistungen bzw. der VAS bezogen, um in Verlauf von Abschnitt 3 ein einheitliches Verständnis über die Anforderungen für Einsatzmöglichkeiten von Wearables zu ermöglichen.

Zunächst zu betrachten ist die Differenzierung zwischen Basisfunktionen, die allen logistischen Lagerprozessen zugrunde liegen, und Zusatzfunktionen, welche wiederum für die Bearbeitung besonderer Anwendungsfälle notwendig werden. Veranschaulicht sind beide Kategorien in Abb. 1.

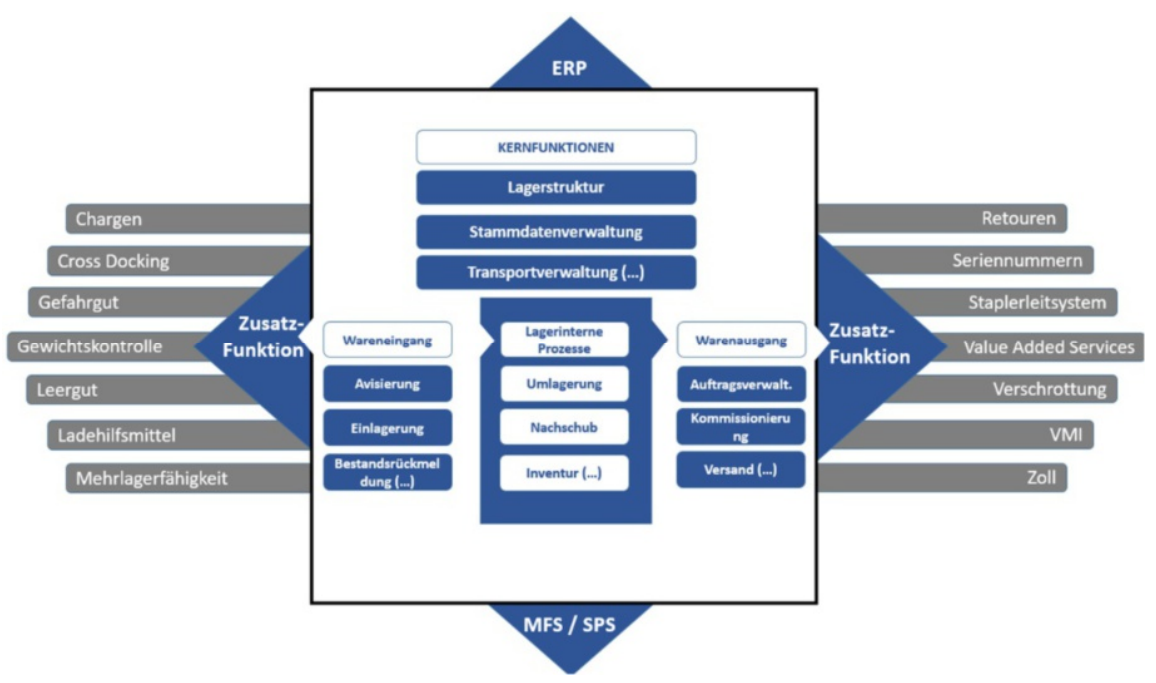

Abb. 1. Warehouse Management Systeme nach VDI-Richtlinie 3601, angelehnt an Fraunhofer IML

Neben den Kern- und Zusatzfunktionen ist dort auf die systemische Eingliederung des Warehouse-Managements anhand der oberen und unteren Rautenspitzen zu erkennen. Zum einen ist dort das führende Auftragsverwaltungssystem aus dem 
Bereich der Enterprise-Ressource-Planning-Systeme (ERP-Systeme). Zum anderen werden Subsysteme im Lager aufgezeigt, welche durch die Materialflusssteuerung $[\mathrm{MFS} / \mathrm{SPS}]$ angedeutet sind. Das Prozesselement VAS findet sich in diesem Modell als eigene Systemfunktionalität im Bereich der Zusatzfunktionen wieder. Demnach muss im Lagerverwaltungssystem eine Logik geschaffen sein, um die VAS funktionslogisch an allen auftretenden Prozesselementen anzuhängen und zu verbuchen. VAS können in zwei unterschiedlichen Ausprägungen auftreten (Klaus et al. 2012; Neumann und Schnöring 2010): entweder ist dies eine Tätigkeit mit Querschnittsfunktion, die im Rahmen einer Standardtätigkeit (Kernfunktion) ausgeübt wird, oder eine Tätigkeit mit komplementärem Charakter, die durch einen zusätzlichen Prozess (Zusatzfunktion) außerhalb der Kernfunktionen abgebildet wird.

Was im konkreten Fall eine Mehrwertdienstleistung darstellt, kann in diesem Kontext hingegen nicht einheitlich definiert werden. In Bezug zum Prozess und der damit verbundenen vertraglichen Ausgestaltung der Leistungsvereinbarung zwischen Kontraktlogistikunternehmen und Kunde, ist von den VAS-Leistungen in den Bereich „Standard“ abhängig. Im Folgenden werden daher einige gängige Beispielprozesse aus der Abwicklung eines Unternehmens in der Fashionlogistik näher betrachtet.

\subsection{VAS in der Kontraktlogistik für die Bekleidungsindustrie}

Der folgende Abschnitt bezieht sich auf das GLASSHOUSE-Pilotprojekt im Umfeld der Fashionlogistik. Im Umfeld der Fashionlogistik stehen vor allem die produkt- und (end-)kundenbezogenen VAS im Fokus, die sich auf die zu versendende Ware beziehen. Dies umfasst sowohl alle Tätigkeiten an Textilen und deren Auszeichnungen und Verpackungen als auch komplementäre Dienstleistungen außerhalb der lagerlogistischen Abwicklung. Diese umfassen die Qualitätssicherung und die Aufbereitung. Diese Prozesse sind zwar in dem Distributionsprozess funktional eingegliedert, stellen aber komplett eigene Prozesslandschaften dar. Diese sind dem Produktions- oder Dienstleistungssektor zuzuordnen, sofern sie nur indirekt mit dem eigentlichen Logistikprozess in Verbindung stehen. Explizit nicht betrachtet werden hingegen alle Dienstleistungen, die nicht in direktem Zusammenhang mit der Bearbeitung einer Textilie stehen. Beispielhaft wären hier die Betreibung eines Customer Service oder die Bereitstellung von Online-Plattformen im Kundenauftrag.

\subsubsection{VAS aus Sicht des Auftraggebers}

VAS sind grundsätzlich in logische Kategorien untergliedert. Das Host-/ERP-System des Kundenunternehmens unterscheidet in der Regel zwischen produktspezifischen und nicht-produktspezifischen VAS.

1) Produktspezifische VAS. In dieser Gruppe sind alle Teile eines Artikels oder einer ganzen Produktlinie gleichartig zu behandeln - unabhängig von ihrer späte- 
ren Verwendung, dem Vertriebskanal oder der Versandart. Die produktspezifischen VAS sind daher häufig bereits im Wareneingang durchzuführen. Dort befinden sich alle Teile eines Artikels gesammelt an einem Ort. Differenzierungen aus Sicht des Kundensystems sind wiederum einzelne Artikel oder Artikelgruppen, die sich über die Verwendung eines gleichen Materials kennzeichnen.

a) Material \& Artikelgruppe. Das Material wird in der Textilindustrie häufig in „Oberstoff“ und „Zutaten“ untergliedert. Oberstoffe bezeichnet dabei das eigentliche Gewebe, aus dem die Textilie gefertigt ist. Zutaten sind alle ergänzenden Komponenten wie Reißverschlüsse, Knöpfe oder Garne. Die materialbezogenen VAS sind häufig für ganze Teilegruppen unterschiedlicher Produkte durchzuführen, die aus gleichen Komponenten oder Materialien gefertigt wurden. Dies geschieht häufig unabhängig von ihrer späteren Verwendung. Typisch hierfür ist bspw. das Aufbereiten von Hängewarenartikeln in einem „Tunnelfinisher“. Diese werden aus einem Karton entnommen, mit einem Kleiderbügel versehen und anschließend mit Dampf behandelt, um Knitterfalten zu glätten.

b) Artikel. Diese Gruppe behandelt das Thema VAS spezifischer als die Kategorie Material. Eine gleichartige Jacke wurde bspw. einmal mit- und einmal ohne Fellkragen produziert. Entsprechend erfolgt eine unterschiedliche Behandlung des Teils. Typisch sind hier das Entfernen von Schonbezügen und Transportsicherungen oder die Aufbereitung von Fellkrägen.

2) Nicht-produktspezifische VAS. In diese Gruppe fallen alle VAS, die erst durch den Distributionsauftrag festgelegt werden. Die spätere Verwendung des Teils, bzw. die Zuteilung legen die Bearbeitungsschritte fest, die jedes Einzelteil zu durchlaufen hat.

a) Kunde. Die Kategorie Kunde repräsentiert die größte Gruppe der Sonderarbeiten. Zunächst muss aus der Sicht des Logistikdienstleisters noch der Begriff Kunde differenziert werden. Kunde, aus der Perspektive des Kontraktlogistikers ist i.d.R. das Textilunternehmen, welches den Logistikpartner mit der Distribution seiner Waren beauftragt. Die Kunden des Textilunternehmens sind wiederum dessen Verkaufspartner (B2B) oder die Endkunden (B2C), die jeweils ihre Ware durch den Logistikdienstleister erhalten. Im Folgenden wird daher folgende begriffliche Abgrenzung vorgenommen:

- Textilit: Textiles Produktionsunternehmen oder Inhabergesellschaft der Textilmarke. Ein Textilit ist nicht notwendigerweise der Produzent einer Textilie, ist jedoch für die Lieferkette von Rohware bis Fertigprodukt verantwortlich.

- Kunde: Der Kunde wird durch das Partnerunternehmen des Textiliten dargestellt. Dieses kann durch einen Online- oder stationären Handel als Verkaufsplattform auftreten, wodurch der Endkunde die Ware erwerben kann.

- Endkunde: Der Endkunde erhält entweder über die Zwischenstation des Zwischenhändlers (Kunde) oder über einen direkten Vertriebskanal des Textiliten das Angebot zum Erwerb der Ware. Sollte eine direkte Beziehung zwischen Textilit und Endkunde vorliegen, z.B. durch Betreiben einer eigenen Online- 
shop-Plattform durch des Textiliten, so werden Endkunden durch den Logistikpartner auch direkt mit Ware beliefert.

Alle drei Kategorien der Dimension „Kunde” stellen eigene Anforderungen an die VAS-Bearbeitung in der Warendistribution. Eine der Besonderheiten dieser Dimension liegt darin, dass immer erst durch die Übertragung eines Kommissionierauftrages eine Zuordnung von Ware zu VAS entsteht. Erst durch die Zuteilung einer Ware zu einem (End-)Kunden durch das Host-System werden auch die VAS dem Einzelteil zugewiesen. Darunter würde bspw. das Anbringen eines kundenindividuellen Preisetiketts (B2B) oder das Einlegen eines individualisierten Anschreibens (B2C) fallen.

b) Versanddienstleister. Außerhalb der Dimension „Kunde“ stellt auch die Versandabwicklung eine Herausforderung für den lagerlogistischen Prozess dar, die z.T. per VAS gesteuert werden. Grundsätzlich unterschieden wird zunächst zwischen Selbstabholern, die einen eigenen Transport organisieren, Speditionsversand und den KEP-Versand (Kurier-Express-Paket-Dienst). Während Speditionen für große Aufträge oder Mengen mit gleicher „Quelle-Ziel-Beziehung“ beauftragt werden, sind KEP-Dienstleister für das kleinteilige Geschäft geeignet. Je nach Versanddienstleister und Versandprodukt sind unterschiedliche Bearbeitungsschritte notwendig. Die Mehrwertdienstleistungen umfassen die Zusammenstellung der Ware, die Wahl der richtigen Versandverpackungen, das Anbringen spezieller Label, die Zollbearbeitung, und die z. T. spezielle Avisierung.

c) Vertriebskanal. Im Bereich Vertriebskanal ergeben sich weitere Gruppierungen, nach denen sich die VAS Bearbeitung zu richten hat. Das Gebiet der Absatzkanäle umfasst aus Sicht des Logistikunternehmens grundsätzlich 3 Kategorien: Omnichannel/Multichannel, B2C und B2B.

Überschneidungen in der Auswirkungsweise der Kategorien sind möglich. Im B2B-Umfeld kann es sich bspw. auch um ein eigenes Filialnetz des Textiliten, einen Onlineshop mit eigenem B2C-Lager und Versand bei einem weiteren Dienstleister handeln. Der Begriff „Omnichannel“ deutet darüber hinaus an, dass auch Querverbindungen zwischen den Kanälen existieren. So kann eine Retoure aus dem Online-Versandhandel ggf. auch in einer Filiale zurückgegeben werden. Für das Portfolio der VAS-Bearbeitung ergeben sich daraus weitere Anforderungen. Im oben gewählten Beispiel werden Retouren eines Endkunden über den B2B-Retourenkanal des Kunden wieder an den Logistikpartner „en bulk“ avisiert und zurückgeschickt. Bereits durchgeführte Sondertätigkeiten wie das Anbringen besonderer Etiketten, müssen rückabgewickelt werden, um das Teil in einen erneut zuteilbaren Zustand zu überführen.

\subsubsection{VAS im lagerlogistischen Prozess: Ausprägungen und Verrichtungsorte}

Aus der Sicht des Auftraggebers wurden verschiedene Kategorien der VAS-Bearbeitung anhand logischer Gruppen gebildet. Diese Gruppierung hilft dem Dienst- 
leister, sinnvolle Verrichtungsorte zu identifizieren. Bereits in Abschnitt 2.2.1 wurde angedeutet, dass es sich anbietet, produktspezifische Tätigkeiten bereits vor/während des Wareneingangsprozesses durchzuführen. Einerseits befinden sich alle gleichartig zu bearbeitenden Teile gesammelt an einem Ort. Andererseits werden dem Dienstleister vertriebskanalabhängige, kundenspezifische oder versandbezogene Tätigkeiten erst mit der Übertragung eines Kommissionierauftrages bekannt. Aus diesem Grund unterscheidet das WMS unterschiedliche Verrichtungsorte für VAS, die wiederum an Prozessschritte geknüpft sind. Einige Fälle von VAS sind systemlogisch zu bearbeiten, sodass im Prozess kein Mitarbeiter eingreifen muss. Diese Fälle sind jedoch zumeist abhängig vom Automatisierungsgrad des Lagers. Zunächst werden folgende Verrichtungsorte/Prozessschritte für die VAS-Bearbeitung unterschieden.

a) Wareneingang. Alle Teile eines Wareneinganges bestehen in der Regel aus dem gleichen Artikel. Mit datentechnischer Avisierung werden zu dem auch zu verrichtende Sondertätigkeiten für den Artikel übertragen. Bspw. können das Entfernen einer Transportverpackung und die geplante Aufbereitung der Ware darunterfallen. Die Ware wird in diesem Schritt für die Vereinnahmung vorbereitet. Dies führt zur vollständigen Bearbeitung des Wareneingangs, bevor die Warenvereinnahmung beginnen kann.

b) Vereinnahmung. Während der Vereinnahmung der Ware werden parallel zur Einzelteilerfassung im WMS Dienstleistungen durchgeführt. Beispielhaft ist hier das Anbringen eines Kleiderbügels, gefolgt von dem Einlesen eines EAN-Barcodes, durch den das Einzelteil identifiziert und gezählt wird. Je nach Anlieferart ist nicht für alle Wareneingänge eine Applikation des Kleiderbügels notwendig. Handelt es sich um hängende, angelieferte Ware, die in liegender Form vereinnahmt werden, ist dies nicht notwendig. Aus diesem Grund stellt das sog. „Aufbügeln" eine Sondertätigkeit dar.

c) Einlagerung. Nur selten sind Einlagerungsprozesse durch zusätzliche VAS gekennzeichnet. Zum Teil werden für die Einlagerung jedoch schon die Vertriebskanäle festgelegt, so dass bestimmte Artikel daten- und lagertechnisch getrennt gelagert werden. Beispielhaft ist hier ein eigener B2C-Bestand, der an einem physisch getrennten Lagerort eingelagert wird. Je nach Funktionalität des WMS ist dabei für den Mitarbeiter keine Abweichung vom Standardprozess feststellbar.

d) Kommissionierung. Während der Kommissionierung $\mathrm{zu}$ beachtende VAS beziehen sich zumeist auf die Planung und Befüllung der Versandeinheiten (Kartons oder Packs). Unterschieden wird hier bspw. bei der Artikelreinheit (nur gleiche Artikel wie Jacken in verschiedenen Größen), SKU-Reinheit (nur die gleiche EAN wie eine Jacke in gleicher Größe und Farbe) oder artikelaufsteigender Planung (wie unterschiedliche Artikel in einem Karton in bestimmter Sortierung).

e) Sonderarbeiten. Direkt im Anschluss an die Kommissionierung eines Artikels werden sämtliche kunden- oder vertriebsprogrammspezifischen Dienstleistungen an der Ware durchgeführt. Dazu werden kommissionierte Behälter in einen eigenen Bereich verfrachtet, in dem geschulte Mitarbeiter die VAS-Bearbeitung systemgestützt vornehmen. Ein Großteil des Arbeitsaufwandes für VAS fällt in 
diesem Prozessschritt an. Klassische Beispiele sind das Anbringen kundenspezifischer Artikeletiketten, das Anbringen von Warensicherungen und das Austauschen von Kleiderbügeln gegen kundenspezifische Bügel. Im Ergebnis dieses Prozesses entstehen für einen bestimmten Kunden präparierte Aufträge, die dem Versandprozess zugesteuert werden. Je nach Textilit und vertraglicher Vereinbarung werden allen kommissionierten Mengen in diesem Schritt zusätzlich einer Einzelteilzählung unterzogen, um das Kommissionierergebnis zu verifizieren.

f) Verpackung. Während des Bearbeitungsschritts „Verpacken“ erhält eine Versandeinheit (Karton oder Pack) entsprechende Versandpapiere. Dazu wird es zunächst gewogen und bei dem hinterlegten Carrier angemeldet. Der Versandpartner stellt daraufhin ein Versandlabel mit Trackinginformationen zur Verfügung. Dieses wird auf der Versandeinheit appliziert. Das WMS erzeugt zusätzlich Lieferschein und Packstückinhaltsliste. Im Schritt Verpacken, wird die Versandeinheit verschlossen. Oftmals ist ein Umpacken notwendig, falls eine Kunden- oder Endkundenspezifische Versandverpackung erforderlich sein sollte. Häufig ist hiervon der Vertriebskanal B2C betroffen. Endkundensendungen sollen ein „unboxing event" ermöglichen und werden daher aufwändig in kleinen Schachteln mit z.T. personalisierten Lieferpapieren verpackt. Alle Tätigkeiten, die nicht für den Versand unbedingt notwendig sind (i.d.R. nur Lieferschein und Versandlabel ausdrucken und anbringen), fallen dabei in den Bereich der VAS. Typisch für diesen Prozess: Waschanweisungen, Gutscheine oder „Giveaways“ beilegen.

g) Warenausgang. Im Warenausgang wird die Versandfähigkeit eines (verpackten) Artikels hergestellt und geprüft. Hierzu werden Richtlinien berücksichtigt bzw. weitere Funktionen erfüllt. Eine nachvollziehbare Dokumentation der auslagernden Ware ist dabei essentiell, die über VAS digitalisiert werden kann. Darüber hinaus fallen die Einhaltung von Qualitätsstandards und die Bereitstellung von Ladepapieren als weitere zu berücksichtigen Faktoren im Warenausgang an. Um einen Warenausgang zu gewährleisten, wird die Ware auf einer definierten Fläche zwischengelagert. Hierzu werden Hilfsmittel wie Hubwagen, Ameisen und Gabelstapler genutzt.

\section{Unterstützung von VAS durch Smart Products und Wearables}

\subsection{Stand der Technik: Wearables im Unternehmen (Fashionlogistik) und am Markt}

Das Unternehmen setzt aktuell vor allem klassische Automatisierungstechnik zur Abwicklung der Intralogistik ein. An Lagerstandorten oder in Teilbereichen ohne hohen Automatisierungsgrad werden hingegen bereits heute mobile Geräte eingesetzt, um verschiedene Prozessschritte nach dem „Mann zur Ware“-Prinzip zu unterstützen (Almeida und Ferreira 2009). Sie setzen dazu hauptsächlich MDE-Geräte (Mobile-Daten-Erfassungs-Geräte) verschiedener Hersteller ein. Mit Hilfe der 
mobilen Datenerfassung werden prozessrelevante Daten ortsungebunden erfasst und dem Warehouse-Management-System zur Verfügung gestellt. Die Informationen werden mit Hilfe mobiler Endgeräte angezeigt und erfasst (Wölfle und Schubert 2006). In der Fashionlogistik kommen dabei zwei verschiedene Typen mobiler Endgeräte zum Einsatz, die sich hauptsächlich durch die Datenerfassung voneinander unterscheiden. Zum einen sind die MDE mit Barcodescanner ausgestattet, zum anderen wird auf RFID-Technik zurückgegriffen. Durch die ortsungebundene Erfassung werden Geschäftsprozesse dahingehend optimiert, dass Zeiten für Informationsgewinnung und -erfassung eingespart werden. Hinzu kommt eine signifikante Verbesserung der Arbeitsergonomie für die Mitarbeiter.

Das Sortiment an Wearables am Markt ist jedoch weitaus vielseitiger. Vor allem in Zusammenhang mit Smart Products wird oftmals von Smart Wearables gesprochen, welche intelligente, tragbare Technologien/Computersysteme darstellen, die am Körper oder am Kopf getragen werden (Karahanoğlu und Erbuğ 2011). Darunter fallen bspw. Smart Watches, Datenbrillen, Activity Tracker bzw. Fitness Tracker oder smarte Kleidung/Schuhe. Die Entwicklung solcher Technologien stieg in den letzten Jahren deutlich an.

Zweck solcher Wearables sind in der Regel die Erfassung zusätzlicher Informationen, die Bereitstellung von Auswertungen und Durchführung von Anweisungen. Somit unterstützen Smart Wearables den Benutzer bei Tätigkeiten in der realen Welt und gewährleisten durch hochentwickelte Sensorik die Erfassung von Daten (Kruse Brandão und Wolfram 2018).

\subsection{Herausforderungen bei der technologischen Unterstützung durch Smart Glasses im Umfeld der VAS}

Bei Betrachtung der technologischen Unterstützung von Smart Glasses im Umfeld der VAS fallen sowohl ergonomische als auch funktionslogische Herausforderungen auf. Viele Smart-Glasses-Anbieter greifen in den technischen Datenblättern neben der Funktionsfähigkeit auch den Aspekt „Fit“" bzw. die Ergonomie auf (Rauschnabel et al. 2015). Um die richtige technologische Unterstützung im Bereich VAS durch Smart Glasses gewährleisten zu können, muss erst festgestellt werden, für welchen Zweck der VAS eingesetzt werden soll. Die Smart Glasses verfügen über ein kleines Display im Blickfeld des Trägers, das verhältnismäßig wenig Informationen gleichzeitig darstellen kann. Die Nutzung eines VAS kann ebenfalls durch eine geringe Einsatzdauer charakterisiert werden. Der SmartGlasses-Träger soll mit einem einzigen Blick auf das Display optimal unterstützt werden, indem bspw. ein Eingabefehler erkannt und mit einer Visualisierung angezeigt wird. Ein VAS kann auch durchgängig in einem Geschäftsprozess in Einsatz genommen werden, wodurch ergonomische Anforderungen eine besonders relevante Rolle spielen. Hierbei kann es sich bspw. um automatisierte Kontrollfunktionen handeln, die in jeden Prozessschritt vom Smart-Glasses-Träger bestätigt werden müssen. Da der Träger der Smart Glasses für einen längeren Zeitraum eine solche Funktion nutzen kann, muss bspw. die richtige Gestaltung des Dis- 
play-Layouts berücksichtigt und eine störfreie Arbeitsweise mit der Hardware gewährleistet werden.

Weiterhin ist der ergonomische Einsatz von Smart Glasses vom Verrichtungsort abhängig. Handelt es sich bspw. um ein gut ausgeleuchtetes und weitflächiges Lager, ist die Nutzung bestimmter Datenbrillen und der VAS nur eingeschränkt sinnvoll. Einige Typen von Displays sind nur in kleinen und dunkleren Umgebungen gut erkennbar, wie bspw. in Lageräumen oder Labors. Der Einsatz der VAS einer Smart Glasses ist ebenfalls für Einsätze im Büro oder entlang eines Produktionsprozesses denkbar (Reif und Günthner 2009).

Die Unterstützung der produktspezifischen und nicht produktspezifischen VAS mithilfe von Smart Glasses im Bereich des Unternehmens der Fashionlogistik stellt ebenfalls eine Herausforderung dar. Während einerseits die produktspezifischen VAS direkt bestimmt werden können, müssen die nicht produktspezifischen erst bei der Distribution geplant werden. Schwierigkeiten bei der realen Umsetzung sind an dieser Stelle denkbar.

\subsection{Einsatzmöglichkeiten von Smart Glasses: Literaturrecherche und Ergebnisse}

In Bezug auf die Einsatzmöglichkeiten der Smart Glasses wurde eine Literaturrecherche nach Webster und Watson durchgeführt, indem konkrete Suchterme in ausgewählten Datenbanken verwendet wurden. Mithilfe einer definierten Sichtungsmethode bestimmter Schlüsselwörter, Abstracts und Inhalte der Fachliteratur, konnten diverse Suchtreffer als relevant eingestuft werden (Webster und Watson 2016). Es folgt eine Übersicht über die verwendeten Suchterme.

Tabelle 1. Ergebnis der Literaturrecherche - Einsatzmöglichkeiten von Smart Glasses

\begin{tabular}{|c|c|c|}
\hline \# & Datenbank & Suchterm \\
\hline 1 & Google Scholar & $\begin{array}{l}\text { (,Smart Glasses” OR „Datenbrille” OR „,Augmented Reality”) AND } \\
\text { (,,Value Added Service” OR ,Value Added Services” OR „VAS”) } \\
\text { AND („Logistik” OR „logistic”) }\end{array}$ \\
\hline 2 & $\begin{array}{l}\text { Science } \\
\text { Direct }\end{array}$ & $\begin{array}{l}\text { („Value Added Service“ OR „Value Added Services“ OR „Value- } \\
\text { Added Service“ OR „Value-Added Services“) AND („logistics“ OR } \\
\text { „Logistik“ NOT „,tele-communication“) }\end{array}$ \\
\hline 3 & $\begin{array}{l}\text { Wiley (Online } \\
\text { Library) }\end{array}$ & $\begin{array}{l}\text { „Value-added services“ AND Logistik OR logistics AND „Smart } \\
\text { Glasses“ }\end{array}$ \\
\hline 4 & EBSCOHost & $\begin{array}{l}\text { „value-added services“ AND Logistik OR logistics AND „Smart } \\
\text { Glasses“ }\end{array}$ \\
\hline 5 & Emerald & $\begin{array}{l}\text { („Value Added Service“ OR „Value Added Services“ OR „Value- } \\
\text { Added Service“ OR „Value-Added Services“) AND („logistic“ OR } \\
\text { „Logistik“) }\end{array}$ \\
\hline
\end{tabular}


Die Recherche hat folgende Ergebnisse erzielt.

Tabelle 2. Ergebnis der Literaturrecherche - Einsatzmöglichkeiten von Smart Glasses

\begin{tabular}{|c|c|c|c|c|}
\hline \multicolumn{5}{|c|}{ Ergebnis (Anzahl Suchtreffer): Einsatzmöglichkeiten von Smart Glasses } \\
\hline Datenbank & Lagerhaltung & Spedition & $\begin{array}{c}\text { Produktion \& } \\
\text { Verpackung }\end{array}$ & $\begin{array}{c}\text { Weitere } \\
\text { Dienstleistungen }\end{array}$ \\
\hline Google Scholar & 1 & 7 & 16 & 16 \\
\hline Science Direct & 6 & 9 & 15 & 14 \\
\hline Wiley & 3 & 4 & 3 & 6 \\
\hline Emerald & 18 & 21 & 27 & 40 \\
\hline Konsol. Anzahl VAS ${ }^{1}$ & 5 & 16 & 13 & 29 \\
\hline Konsol. Anzahl SG-VAS ${ }^{2}$ & 5 & 9 & 8 & 20 \\
\hline
\end{tabular}

Auf Basis der etablierten Quellen folgt Tabelle 3, die die potenziellen Einsatzmöglichkeiten von VAS in der Logistik kategorisiert. Die Kern- und Zusatzfunktion beziehen sich hierbei auf die Beschreibung aus Abschnitt 2.1.

Tabelle 3. VAS - Einsatzmöglichkeiten von Smart Glasses

\begin{tabular}{|c|c|c|c|}
\hline \multicolumn{4}{|c|}{ Lagerhaltung VAS } \\
\hline \# & Einsatzmöglichkeiten & Einsatz von Smart Glasses & $\begin{array}{l}K F / \\
Z F^{3}\end{array}$ \\
\hline 1 & Lagerung & $\begin{array}{l}\text { Die Lagerung von Produkten kann unterstützt werden } \\
\text { durch den Einsatz von Smart Glasses. Hierdurch können } \\
\text { zum Beispiel bei der Platzierung von Gütern wichtige In- } \\
\text { formation geliefert werden, was in welcher Menge wo ge- } \\
\text { lagert werden soll. }\end{array}$ & KF \\
\hline 2 & $\begin{array}{l}\text { Lagerauffüllung, } \\
\text { elektronisch-automati- } \\
\text { sierte Lagerauffüllung }\end{array}$ & $\begin{array}{l}\text { Mithilfe von Smart Glasses ist es möglich, den Lagerbe- } \\
\text { stand aufzufüllen. Zudem können Informationen zum ak- } \\
\text { tuellen Lagerbestand aufgerufen werden. }\end{array}$ & KF \\
\hline 3 & $\begin{array}{l}\text { Lager-Dienstleistungen, } \\
\text { Zolllager als auch } \\
\text { Speziallager- } \\
\text { dienstleistungen }\end{array}$ & $\begin{array}{l}\text { Smart Glasses sind ein Hilfsmittel, welches den Benutzer } \\
\text { bei der Verarbeitung von Informationen unterstützt. So- } \\
\text { wohl Speziallager als auch Zolllager verlangen eine be- } \\
\text { stimme Art der Lagerhaltung. Sowas wie Zolldeklaratio- } \\
\text { nen, -vorlagen, -anmeldungen und -abfertigungen kann } \\
\text { mittels Smart Glasses unterstützt bzw. verrichtet werden. } \\
\text { Die Betrachtung und Vorlagen für solche Arten von La- } \\
\text { gern kann mittels Smart Glasses erfolgen. }\end{array}$ & ZF \\
\hline 4 & \begin{tabular}{|l} 
Zentralisierung der \\
Lagerbestände
\end{tabular} & $\begin{array}{l}\text { Die Betrachtung verschiedener Layouts für zentralisierte } \\
\text { Lagerstände kann per Smart Glasses durchgeführt werden. }\end{array}$ & ZF \\
\hline 5 & Ersatzteillogistik & $\begin{array}{l}\text { Durch Betreiben eines Ersatzteillagers wird ein VAS ge- } \\
\text { neriert. Dieses kann durch Smart Glasses optimiert wer- } \\
\text { den. Dadurch können Schritte beim Einlagern, wie z. B. } \\
\text { Produktart, -menge, -platzierung, -lagerung, erleichtert } \\
\text { werden. }\end{array}$ & ZF \\
\hline
\end{tabular}

1 Konsolidierte Anzahl an allgemeinen VAS.

2 Konsolidierte Anzahl an VAS für Smart Glasses.

$3 \mathrm{KF}=$ Kernfunktion $/ \mathrm{ZF}=$ Zusatzfunktion. 


\begin{tabular}{|c|c|c|c|}
\hline \multicolumn{4}{|c|}{ Spedition VAS } \\
\hline \# & Einsatzmöglichkeiten & Einsatz von Smart Glasses & $K F / Z F$ \\
\hline 1 & Transport & $\begin{array}{l}\text { Smart Glasses können den Lieferanten via Navigationssys- } \\
\text { tems unterstützen, um so Informationen der aktuellen Route } \\
\text { auf dem Display auszugeben. }\end{array}$ & KF \\
\hline 2 & $\begin{array}{l}\text { Wunschzeitenliefe- } \\
\text { rung } 4\end{array}$ & $\begin{array}{l}\text { Smart Glasses können dazu dienen, dem Lieferanten anzu- } \\
\text { zeigen, wann welche Lieferungen über welche Routen getä- } \\
\text { tigt werden sollen. }\end{array}$ & KF \\
\hline 3 & $\begin{array}{l}\text { Sendungsverfolgung } \\
\text { (Track and Tracing) }\end{array}$ & $\begin{array}{l}\text { Die Verfolgung der Sendung mittels Smart Glasses zur Be- } \\
\text { stimmung des Standortes ist möglich. }\end{array}$ & $\mathrm{ZF}$ \\
\hline 4 & $\begin{array}{l}\text { Flottenmanagement/ } \\
\text { Operationen }\end{array}$ & $\begin{array}{l}\text { Flottenmanagement ist unterstützbar. Information sind via } \\
\text { Display einer Datenbrille einsehbar. }\end{array}$ & $\mathrm{ZF}$ \\
\hline 5 & Ladungsverarbeitung & $\begin{array}{l}\text { Die Ladungsverarbeitung, Annahme und Verfrachtung, } \\
\text { kann mittels Smart Glasses beschleunigt werden. So können } \\
\text { zum Beispiel Kontrollen und Bestätigungen der Produkte } \\
\text { mittels Smart Glasses erfolgen. }\end{array}$ & $\mathrm{KF}$ \\
\hline 6 & Rollende Fabriken ${ }^{5}$ & $\begin{array}{l}\text { Rollende Fabriken können mittels Smart Glasses kontrolliert } \\
\text { werden. So kann der Empfänger die Ware kontrollieren und } \\
\text { z. B. Kontakt mit dem Logistikunternehmen aufnehmen. }\end{array}$ & $\mathrm{KF}$ \\
\hline 7 & Cross-Docking & $\begin{array}{l}\text { Cross-Docking Prozesse können durch Smart Glasses wahr- } \\
\text { genommen werden. Somit entfallen Lagerhaltungskosten. } \\
\text { Es ist möglich, durch die Datenbrillen diese Cross-Docking } \\
\text { Prozesse zu leiten und unterstützen. Mittels Smart Glasses } \\
\text { lassen sich Waren direkt annehmen und den darauffolgen- } \\
\text { den Transport zum Kunden zu planen. }\end{array}$ & $\mathrm{ZF}$ \\
\hline 8 & $\begin{array}{l}\text { Multimodaler } \\
\text { Transport }\end{array}$ & $\begin{array}{l}\text { Smart Glasses können den Nutzer helfen bei der Koordinie- } \\
\text { rung und Konsolidierung verschiedener Transportmittel. }\end{array}$ & $\mathrm{ZF}$ \\
\hline 9 & $\begin{array}{l}\text { Lieferung mittels Ein- } \\
\text { satzes von Robotern/ } \\
\text { Drohnen, selbstfah- } \\
\text { renden Systemen wie } \\
\text { fahrerlosen Transport- } \\
\text { systemen }\end{array}$ & $\begin{array}{l}\text { Die Kontrolle und die Steuerung der Lieferung kann mithil- } \\
\text { fe von Robotern wie bspw. Drohnen durchgeführt werden, } \\
\text { welche von Smart Glasses gesteuert werden. }\end{array}$ & $\mathrm{ZF}$ \\
\hline
\end{tabular}

\begin{tabular}{|c|c|c|c|}
\hline \multicolumn{4}{|c|}{ Produktion \& Verpackung VAS } \\
\hline \# & Einsatzmöglichkeiten & Einsatz von Smart Glasses & $K F / Z F$ \\
\hline 1 & $\begin{array}{l}\text { Verpackungsdienst- } \\
\text { leistungen / } \\
\text { Verpackung: (z.B. } \\
\text { Standardverpackung, } \\
\text { Dreieckstuben oder } \\
\text { Kartons, etc.) }{ }^{6}\end{array}$ & $\begin{array}{l}\text { Smart Glasses können dem Nutzer bei Verpackungsdienst- } \\
\text { leistungen unterstützen. Um Produkte zu verpacken, muss } \\
\text { man das zu verwendete Verpackungsmaterial sowie die } \\
\text { Verpackungsart bzw. die Verpackungsprozedur, nach einer } \\
\text { Vorlage wie zu verpacken ist, befolgen. Smart Glasses lie- } \\
\text { fern wichtige Informationen, mit welchen Verpackungen } \\
\text { verpackt werden soll und wie diese zu verpacken sind. }\end{array}$ & $\mathrm{KF}$ \\
\hline
\end{tabular}

4 Just-In-Time (JIT) Lieferung, Terminvereinbarung der Lieferung Same-Day-Delivery, 90 Min. Delivery, Expresslieferung, On-Demand Transport, Direkttransport.

5 Auch in Bezug auf die Kategorie Produktion und Verpackung.

6 Umweltfreundliche Verpackung, Geschenkpapier, kundenspezifische Verpackung, Verpackungsveredelung, landesspezifische Verpackung: Exportverpackungen) Umpacken von Retouren und Verkauf, Rekonditionierung, Schrumpffolie, Umverpackung, Ausoder Ein- oder Verpacken, Co-Packing. 


\begin{tabular}{|c|c|c|c|}
\hline 2 & $\begin{array}{l}\text { Etikettierung: Umeti- } \\
\text { kettierung, Kenn- } \\
\text { zeichnung, Labeling, } \\
\text { Preisauszeichnung, } \\
\text { Barcodierung }\end{array}$ & $\begin{array}{l}\text { Durch Smart Glasses ist es möglich, die Etikettierung zu } \\
\text { vereinfachen, indem Informationen geliefert werden, wel- } \\
\text { ches Produkt wie etikettiert werden soll. }\end{array}$ & KF \\
\hline 3 & $\begin{array}{l}\text { Gruppierung bzw. pa- } \\
\text { lettisieren von Gütern, } \\
\text { Modulbildung, Bau- } \\
\text { gruppen- } \\
\text { zusammenstellung, } \\
\text { Kitting, Produkthand- } \\
\text { ling, Setbildung }\end{array}$ & $\begin{array}{l}\text { Um Güter Gruppen zu erstellen, ist es wichtig zu wissen, } \\
\text { welche Produkte gruppiert werden sollen. Smart Glasses } \\
\text { bieten so eine Information. }\end{array}$ & KF \\
\hline 4 & $\begin{array}{l}\text { Fertigung/Herstellung, } \\
\text { Konfektionierung [se- } \\
\text { rienmäßige Herstel- } \\
\text { lung- Anm. d. Verf.], } \\
\text { Produkten, Auf- } \\
\text { tragserfüllung, etc. }^{7}\end{array}$ & $\begin{array}{l}\text { Smart Glasses können den Prozess der Produktion unterstüt- } \\
\text { zen, indem diese dem Nutzer Informationen über Arbeitsab- } \\
\text { läufe und Arbeitsprozesse liefert. }\end{array}$ & $\mathrm{KF}$ \\
\hline 5 & $\begin{array}{l}\text { Montage, Vormonta- } \\
\text { ge, Endmontage, Pro- } \\
\text { duktmontage, Fracht- } \\
\text { montage, leichte Pro- } \\
\text { duktionstätigkeiten }\end{array}$ & $\begin{array}{l}\text { Um Produkte auf richtige Art und Weise zu fertigen, ist es } \\
\text { wichtig, wie diese montiert werden müssen. Diese Informa- } \\
\text { tion kann durch die Nutzung von Smart Glasses gewonnen } \\
\text { werden. }\end{array}$ & KF \\
\hline 6 & $\begin{array}{l}\text { Konfiguration bspw. } \\
\text { in Bezug auf die Ver- } \\
\text { packung wie z.B. } \\
\text { Smartphone- } \\
\text { Verpackungen }\end{array}$ & $\begin{array}{l}\text { Es können Informationen via Smart Glasses eingesehen } \\
\text { werden, die wichtig zur Konfigurierung/Einstellung des } \\
\text { Produktes sind. }\end{array}$ & $\mathrm{ZF}$ \\
\hline 7 & 3D-Druck & $\begin{array}{l}\text { Mithilfe von Smart Glasses ist es möglich, den 3D-Druck zu } \\
\text { realisieren. Die Steuerung und Kontrolle erfolgt ebenfalls } \\
\text { über die Smart Glasses. }\end{array}$ & $\mathrm{ZF}$ \\
\hline 8 & Sortierung & $\begin{array}{l}\text { Datenbrillen können den Nutzer bei der Sortierung von Gü- } \\
\text { tern unterstützen, die benötigten Informationen findet man } \\
\text { im Display der Smart Glasses. }\end{array}$ & $\mathrm{ZF}$ \\
\hline
\end{tabular}

Nachdem eine Kategorisierung in Bezug auf die VAS in der Logistik vorgenommen werden konnte, kann die Etablierung von weiteren Dienstleistungen erhoben werden. Diese sind der Tabelle 3 nicht beigefügt, da sie breit gefächert sind und sich in originär logistische- sowie in originär nicht-logistische Aktivitäten zuordnen lassen. Die Begriffe wurden in Abschnitt 2 differenziert. Der Zweck solcher Aktivitäten umfasst vor allem wertschöpfende Dienstleistungen. Unter die logistischen Aktivitäten fallen bspw. Bestandskontrollen/Inventur, Retourenbearbeitung und Qualitätskontrollen, während den nicht-logistische Aktivitäten bspw. Überwachung von CO2-Emissionen, Kundenbetreuung und Wartungen/Reparaturen zugeordnet werden können. Aufgrund der aufgelisteten Kategorien und unter Berücksichtigung der entsprechenden Kern- oder Zusatzfunktion ist es denkbar,

7 Co-Manufacturing, Bearbeitung, Aufbereiten von Waren, Verarbeitung, Endverarbeitung z. B. Mischen von Aromen, Waschen, Veredelung. 
dass viele Anwendungsfälle für Smart Glasses in der Realität umsetzbar sind. Durch die Nutzung der Smart Glasses in den oben beschriebenen Tätigkeiten können viele Kern- und Zusatzfunktionen optimiert und an das Prozesslayout eines Unternehmens angepasst werden.

\section{Zusammenfassung und Ausblick}

Nach der Abgrenzung klassischer logistischer Sondertätigkeiten mit den durch Smart Glasses unterstützten VAS, konnte mithilfe einer systematischen Literaturrecherche eine Kategorisierung vorgenommen werden. Diese stützt sich auf logistische Einsatzmöglichkeiten für Smart Glasses, in denen VAS besonders zu tragen kommen. Durch die Ausführung der logistischen Anwendungsfälle von VAS eines Unternehmens aus der Fashionbranche wird dadurch nicht nur ein Grundverständnis über die Bedeutung von VAS gebildet, sondern die Adaption von VAS auf weitere Wearables entlang der Supply Chain eines Unternehmens sensibilisiert. Innerhalb der vorgestellten Kategorisierung können Herausforderungen und Unterstützungen durch den Einsatz von Smart Glasses und dessen Auswirkung auf VAS hervorgehoben werden. Dadurch kann die Analyse in Bezug auf technologischer Verbesserung von bestehenden Systemen vorangetrieben werden. Die besondere Relevanz dieses Beitrags zeichnet sich durch den Bedarf an Diversifikation zwischen Logistikdienstleistern in der Praxis aus. Während der Fokus auf gutem Preis-Leistungsverhältnis eines Logistikdienstleisters tendenziell sinkt, steigt der Bedarf an weitreichenden Zusatzlösungen, um sich am Markt hervorzuheben (Davies et al. 2006). Somit stellt der Beitrag individualisierte VAS vor, um einen Mehrwert in logistische Prozesse zu schaffen. Da dieser Mehrwert durch den Einsatz von modernen Wearables ermöglicht wird, ist sowohl mit technischen- als auch ergonomischen Herausforderungen zu rechnen. Eine reelle Umsetzung könnte somit herausfordernd sein. Eine nicht ausgereifte Hardware der Wearables kann hierzu als Auslöser herangezogen werden. Mangelnde Langzeitbeobachtungen und unzureichende Referenzen aus der Praxis können ebenfalls zu Besorgnis von Unternehmen führen. Ein nutzeneffektiver VAS mithilfe von smarten Wearables wie Smart Glasses, ist somit ebenfalls nicht garantiert.

Jedoch kann im Zeitalter der Industrie 4.0 ein schnellwachsender technologischer Fortschritt beobachtet werden. Der Begriff „Industrie 4.0“ beschreibt die Evolution von Mechanisierung, über Massenfertigung und der digitalen Revolution durch elektronischen Einsatz bis hin zur digitalen Vernetzung von Menschen und Maschinen (Elbestawi et al. 2018). Dieses Netz der Mensch-Maschinen-Kommunikation birgt viele technologische Forschungsansätze und treibt den Reifegrad von intelligenten Produkten (Smart Products) ebenfalls voran. Dadurch kann die Entwicklung weiterer Anwendungsfälle für smarte Wearables und die Erbringung von VAS entlang der Supply Chain eines Unternehmens prognostiziert werden. 


\section{Literatur}

Almeida DLM de, Ferreira JCE (2009) Analysis of the Methods Time Measurement (MTM) Methodology through its Application in Manufacturing Companies. In: Flex Automation Intelligent Manufactoring. FAIM, United Kingdom, Middlesbrough

Bongartz A (2018) Der Einfluss der Kundenzufriedenheit auf den Unternehmenserfolg: Die Wirkung von Value Added Services. EIKV-Schriftreihe zum Wissens- und Wertemanagement 31:1-85

Davies A, Brady T, Hobday M (2006) Charting a Path Toward Integrated Solutions. MIT Sloan Management Review 47(3):39-48

Ehrmann H (2017) Logistik. NWB, Herne, 9. Auflage

Elbestawi M, Centea D, Singh I, Wanyama T (2018) SEPT Learning Factory for Industry 4.0 Education and Applied Research. Procedia Manuf 23:249-254

Fraunhofer-Institut (2006) Logistik entdecken. Fraunhofer-Institut für Materialfluss und Logistik

Frohn J (2006) Mehrwertleistungen in der Kontraktlogistik. Dissertation, Universität St. Gallen

Huth M (2015) Wiley-Schnellkurs Logistik. Wiley-VCH, Weinheim

Karahanoğlu A, Erbuğ Ç (2011) Perceived qualities of smart wearables: Determinants of user acceptance. DPPI'11 - Desigining Pleasurable Products and Interfaces. doi: $10.1145 / 2347504.2347533$

Klaus P, Krieger W, Krupp M (2012) Management logistischer Netzwerke und Flüsse. Gabler Verlag, Wiesbaden

Kruse Brandão T, Wolfram G (2018) „Personal Mobile Devices”. In: Kruse Brandão T, Wolfram G (Hrsg) Digital Connection: Die bessere Customer Journey mit smarten Technologien - Strategie und Praxisbeispiele. Springer Fachmedien, Wiesbaden

Laakmann K (2018) «Value-Added Services» als Profilierungsinstrument im Wettbewerb. Peter Lang GmbH, Bern

Meffert H (1994) Marketing-Management. Springer Fachmedien, Wiesbaden

Neumann T, Schnöring M (2010) Erfolgsfaktoren von Value Added Services, in Marketing von Solutions: Innovative Ansätze und Best Practices. Gabler Verlag, Wiesbaden

Rauschnabel PA, Brem A, Ro YK (2015) Augmented reality smart glasses: definition, conceptual insights, and managerial importance. University Michigan-Dearborn

Reif R, Günthner WA (2009) Pick-by-vision: augmented reality supported order picking. Vis Comput 25:461-467. https://doi.org/10.1007/s00371-009-0348-y

Webster J, Watson RT (2002) Analyzing the Past to Prepare for the Future: Writing a Literature Review. MIS Quarterly 26(2):xiii-xxiii

Wölfle R, Schubert P (2006) Prozessexzellenz mit Business Software. Carl Hanser Verlag, München

Zimmerlich A, David D, Veddern MW (2005) Übersicht B2B-Marktplätze im Internet: Branchenspezifische B2B-Marktplätze - empirische Erhebung. Arbeitsberichte des Kompetenzzentrums Internetökonomie und Hybridität 28:1-34 